\title{
Analytical Determination of Optimal Actuators Position for Single Mode Active Reduction of Fixed-free Beam Vibration Using the Linear Quadratic Problem Idea
}

\author{
E. ŻOŁOPA ${ }^{a}$ AND A. BRAŃSKI ${ }^{b, *}$ \\ ${ }^{a}$ Halszki 31/12, 30-611 Kraków, Poland \\ ${ }^{b}$ Laboratory of Acoustics, Department of Electrical and Computer Engineering, Rzeszów University of Technology, \\ Wincentego Pola 2, 35-959 Rzeszów, Poland
}

\begin{abstract}
An analytical solution is obtained, based on linear quadratic problem well-known in the control theory. The problem is formulated for fixed-free beam vibration (fourth order partial differential equation) in Hilbert space and the point control and distributed output is considered. Beam deflection at any point is chosen as a criterion of optimization. In this case it means the linear quadratic problem. Up to now, the linear quadratic problem was formulated many times, but only for the time-dependent equation. The aim of the paper is to obtain the value of the cost functional formulated as the function of distribution of actuators. The minimum of this function leads to the optimal actuators location. The results obtained with this method confirm the results obtained in heuristic way and pure analytical one for separate mode; it is pointed out that the actuators ought to be bonded on the beam sub-regions in which the mode curvatures take their local maximums and the highest value.
\end{abstract}

DOI: 10.12693/APhysPolA.125.A-155

PACS: 43.40.Tm, 43.40.Vn, 77.84.Dy, 77.65.-j, 46.40.-f, 46.40.-f, 62.25.Jk

\section{Introduction}

The $p$-reduction is possible for separate mode and it is realized with actuators [1-4]. The quantity of the reduction depends on many factors [5-10]; they are enumerated in [3]. It seems that the most important is an optimal distribution of actuators on the structure $[5,6]$. A lot of optimization techniques are distinguished; an excellent survey of them may be found in [11] and is repeated in [3]. Two main approaches to this problem are classified. In one of these approaches optimization criteria depend on the choice of controllers/control and in this case the quadratic cost functional of the measure error and the control energy (cf. e.g. [12]) is taken into account. This is the approach nearest to the considered in this article.

In the quoted references, it was not provided the actuators distribution in explicit; only general rules (criteria) were formulated. However, considering effectiveness of the $p$-reduction as the criterion, this problem has been already solved. First, based on the heuristic observations $[1,13]$, it was deduced that the most effective distribution of actuators was on the structure sub-domains with the largest curvatures and the highest value of the curvature; such distribution is called the quasi-optimal (QO) one. Next, this result was confirmed in analytical way [2]. Since then, such distribution may be regarded as optimal $(\mathrm{O})$ one. It is worth stressing that even one actuator bonded anywhere on the beam provides $p$-reduction but the effectiveness is poor [1].

*corresponding author; e-mail: abranski@prz.rzeszow.pl
In the paper, based on linear quadratic (LQ) problem formulated in the control theory [14], an analytical solution of the above problem is also achieved. The boundary problem is formulated for beam vibration in an abstract Hilbert space and is considered the point control and distributed output. As a criterion, minimization of beam deflection at any point is chosen. In this case it means the optimization LQ problem. To our knowledge, up to now, the LQ problem was formulated many times, e.g. in $[11,12,15]$, but for time-dependent equation.

The aim of the present paper is to find the value of the (quadratic cost) functional for the special control $f_{t}$ and then to derive the minimum of the function. Finally it leads to the optimal distribution of actuators. As can be seen, the aim is neither finding an optimal control nor an optimal regulator.

The results obtained with this method confirm the results obtained on the analytical way for separate mode presented in $[2,4]$. To the authors' knowledge, such problem has not been considered yet.

\section{Beam forced vibration with damping and $p$-reduction by actuators}

This theory is repeated after papers $[3,4]$. Let the beam be clamped at one side; geometrical data of the beam are: $\ell$ - length, $S$ - area of the cross-section, $q_{E}=$ $q_{E}(x, t)$ - distributed load force. The beam vibration equation is

$$
E J\left(D_{x}^{4} u+\mu D_{x}^{4}\left(D_{t} u\right)\right)+\rho S D_{t}^{2} u=-q_{E},
$$

where $u=u(x, t)$ is the beam deflection at the point $x$ and the moment $t, E$ is the Young modulus, $J$ is the surface moment of inertia of the beam cross-section, $\rho$ is the mass density, $\mu$ is the internal damping factor, $D_{x}^{4}(\cdot)=\partial^{4}(\cdot) / \partial x^{4}$, and $D_{t}(\cdot)=\partial(\cdot) / \partial t$. 
The boundary conditions are described by the following equations:

$$
\begin{aligned}
& u(x=0, t)=0, \quad D_{x} u(x=0, t)=0, \\
& D_{x}^{2} u(x=\ell, t)=0, \quad D_{x}^{3} u(x=\ell, t)=0 .
\end{aligned}
$$

Besides, initial conditions are assumed to be equal to zero, i.e. $\boldsymbol{u}_{0}=\left\{u(0, x)=0, D_{t} u(0, x)=0\right\}$. The solution of the formulated problem represents forced harmonic vibrations with damping. Let the lateral load force $q_{E}(x, t)=q_{E}(x) \exp \left(\mathrm{i} \omega_{q} t\right)$, where $\omega_{q}$ is the angular frequency. The solution of the above problem, for the steady-state case, can be proposed, after [3, 4] as

$$
u(x, t)=X(x) \exp \left(\mathrm{i} \omega_{q} t\right),
$$

where

$$
\begin{gathered}
X(x)=\sum_{\nu} C_{\nu} X_{\nu}(x)=\sum_{\nu} X_{q ; \nu}(x), \\
\nu=1,2, \ldots \infty,
\end{gathered}
$$

where $C_{\nu}$ are certain constants, $X_{\nu}(x)$ are $\nu$-modes (eigenfunctions), and

$$
X_{\nu}(x)=K_{1}\left(\lambda_{\nu} \ell\right) K_{2}\left(\lambda_{\nu} x\right)-K_{4}\left(\lambda_{\nu} \ell\right) K_{3}\left(\lambda_{\nu} x\right),
$$

where $\left\{K_{1}(z), K_{2}(z), K_{3}(z), K_{4}(z)\right\}$ are the Krylov functions [3,4] and, $\left\{\lambda_{\nu}\right\}$ is a set of eigenvalues: $\left\{\lambda_{\nu} \ell\right\}=$ $\{1.8751,4.6941, \ldots,(2 \nu-1) \pi / 2\}$. To obtain $X_{\nu}(x)$ given by Eq. (6), boundary conditions given by Eqs. (2) and (3) have been used.

The constants $C_{\nu}$ are expressed by

$$
\begin{aligned}
& C_{\nu}=\frac{1}{\left(1+\mathrm{i} \mu \omega_{q}\right) \omega_{\nu}^{2}-\omega_{q}^{2}} D_{\nu}=\frac{1}{\alpha_{\nu}^{2}} D_{\nu}= \\
& \frac{1}{\alpha_{\nu}^{2}} \frac{1}{\rho S} \frac{1}{\beta_{\nu}^{2}} I_{\nu ; E}=C_{\nu}^{*} I_{\nu ; E}, \\
& C_{\nu}^{*}=\frac{1}{\alpha_{\nu}^{2}} \frac{1}{\rho S} \frac{1}{\beta_{\nu}^{2}}, \quad I_{\nu ; E}=-\int_{0}^{\ell} q_{E} X_{\nu}(x) \mathrm{d} x, \\
& \omega_{\nu}^{2}=\frac{E J}{\rho S} \lambda_{\nu}^{4}, \quad \beta_{\nu}^{2}=\int_{0}^{\ell} X_{\nu}^{2}(x) \mathrm{d} x .
\end{aligned}
$$

The problem of the beam vibration with damping and, excitation given by $q_{E}(x, t)$ is thus solved.

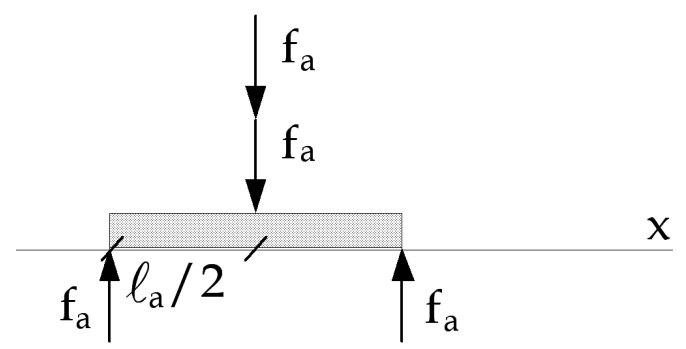

Fig. 1. Interaction actuator-beam.

Adding an effect of actuators on the beam in active vibration reduction, the total load is the sum of $q_{E}(x, t)$ and the forces interacting between actuators and the beam, Fig. 1, and is given by [2-4]:

$$
\begin{aligned}
& f(x)=-q_{E}+\sum_{a}\left[f_{a} \delta\left(x-x_{1 a}\right)-2 f_{a} \delta\left(x-x_{a}\right)\right. \\
& \left.\quad+f_{a} \delta\left(x+x_{2 a}\right)\right],
\end{aligned}
$$

where $x_{1 a}=x_{a}-\ell_{a} / 2, x_{2 a}=x_{a}+\ell_{a} / 2, x_{a}$ is the location of the actuator center (expression in the bracket is the sum of interacting actuators-beam forces), $\delta(\cdot)$ is Dirac delta distribution, $q_{E}$ is the amplitude of the external distributed force, and $\omega_{q}$ is replaced by $\omega_{f}$. For simplicity, all actuators are assumed to be identical from the geometrical and technical point of view. In this case, instead of $I_{\nu ; E}$ in Eq. (8), for $f(x)$ one has

$$
\begin{aligned}
I_{\nu} & =-\int_{0}^{\ell} f(x) X_{\nu}(x) \mathrm{d} x=-q_{E} \int_{0}^{\ell} X_{\nu}(x) \mathrm{d} x \\
& +\sum_{a} f_{a}\left[X_{\nu}\left(x_{1 a}\right)-2 X_{\nu}\left(x_{a}\right)+X_{\nu}\left(x_{2 a}\right)\right] .
\end{aligned}
$$

The expression in square brackets constitutes, in an approximate manner, the second-order central finite difference. Since the distance between nodes $\ell_{a}$ is constant, then the difference can be transformed approximately into

$$
\begin{aligned}
& \frac{1}{\ell_{a}^{2}}\left[X_{\nu}\left(x_{1 a}\right)-2 X_{\nu}\left(x_{a}\right)+X_{\nu}\left(x_{2 a}\right)\right] \approx D^{2} X_{\nu}\left(x_{a}\right) \\
& \quad \approx \kappa_{\nu}\left(x_{a}\right),
\end{aligned}
$$

where $\kappa_{\nu}\left(x_{a}\right)$ is the curvature of the mode $X_{\nu}(x)$ at the point $x=x_{a}[2-4]$.

An approximation carried out in (11) is a consequence of replacement of the differential quotient by the derivative and moreover, the curvature is equal the second derivative in approximately, for small vibrations.

Substituting Eq. (11) into Eq. (10), one obtains

$$
\begin{gathered}
I_{\nu}=-q_{E} \int_{0}^{\ell} X_{\nu}(x) \mathrm{d} x+\sum_{a} f_{a} \ell_{a}^{2} \kappa_{\nu}\left(x_{a}\right)= \\
-I_{q ; \nu}+\sum_{a} p_{a} \kappa_{\nu}\left(x_{a}\right), \quad p_{a}=f_{a} \ell_{a}^{2} .
\end{gathered}
$$

Substituting Eq. (8) into Eq. (5) via Eq. (12), one obtains

$$
\begin{aligned}
& X_{f}(x)=\sum_{\nu} C_{\nu}^{*} I_{\nu} X_{\nu}(x)=\sum_{\nu} C_{\nu}^{*} \\
& \quad \times\left(-I_{q ; \nu}+\sum_{a} p_{a} \kappa_{\nu}\left(x_{a}\right)\right) X_{\nu}(x),
\end{aligned}
$$

and the reduction of vibrations is given by Eq. (4), where $X(x) \equiv X_{f}(x)$.

\section{LQ problem formulation}

To achieve the paper's aim, new variables are introduced. Let assume, that total load can be written as $f(x, t)=f_{x}(x) f_{t}(t)=f_{x} f_{t}$. Let introduce new variables, i.e.

$$
z_{1}=u, \quad z_{2}=D_{t} u,
$$

where $z_{1}=z_{1}(x, t), z_{2}=z_{2}(x, t)$.

Substituting Eq. (14) into Eq. (1), one obtains equations in a matrix form ( $I$ is the identity operator): 


$$
\begin{aligned}
& {\left[\begin{array}{c}
D_{t} z_{1} \\
D_{t} z_{2}
\end{array}\right]=\left[\begin{array}{cc}
0 & I \\
-\frac{1}{\rho S} E J D_{x}^{4} & -\frac{1}{\rho S} E J \mu D_{x}^{4}
\end{array}\right]\left[\begin{array}{l}
z_{1} \\
z_{2}
\end{array}\right]} \\
& +\left[\begin{array}{c}
0 \\
-\frac{1}{\rho S} f_{x}(x)
\end{array}\right] f_{t}(t) .
\end{aligned}
$$

To formally formulate the LQ problem, the boundary problem described in the above subsection should be expressed in the form of an abstract model. For this purpose, one introduces an abstract Hilbert state space $\mathcal{H}:=L^{2}(0, \ell) \oplus L^{2}(0, \ell)$. In this space, the scalar product is defined as $\langle z, w\rangle_{\mathcal{H}}=\left\langle z_{1}, w_{1}\right\rangle_{L^{2}(0, \ell)}+\left\langle z_{2}, w_{2}\right\rangle_{L^{2}(0, \ell)}$. For fixed time $t \geq 0$, the state vector $z(t)=\left[z_{1}(\cdot, t), z_{2}(\cdot, t)\right]^{T}$ belongs to space $\mathcal{H}$. So, the set Eq. (15) may be formulated in abstract form as

$$
D_{t} z(t)=A z(t)+B w(t), \quad y(t)=C z(t) .
$$

The state operator $A:(D(A) \subset \mathcal{H}) \rightarrow \mathcal{H}$ is defined as

$$
A\left[\begin{array}{l}
z_{1} \\
z_{2}
\end{array}\right]=\left[\begin{array}{c}
z_{2} \\
-\frac{1}{\rho S} E J D_{x}^{4} z_{1}-\frac{1}{\rho S} E J \mu D_{x}^{4} z_{2}
\end{array}\right],
$$

where

$$
\begin{gathered}
D(A)=\left\{z \in \mathcal{H}: D_{x} z \in L^{2}(0, \ell), D_{x}^{2} z \in L^{2}(0, \ell),\right. \\
D_{x}^{3} z \in L^{2}(0, \ell), D_{x}^{4} z \in L^{2}(0, \ell), z(0)=0, \\
\left.D_{x} z(0)=0, D_{x}^{2} z(\ell)=0, D_{x}^{3} z(\ell)=0\right\} .
\end{gathered}
$$

The control vector $B$ takes the form

$$
B=\left[\begin{array}{c}
0 \\
-\frac{1}{\rho S} f_{x}(x)
\end{array}\right] \notin \mathcal{H} .
$$

The output operator is $C z=z_{1}$. One observes the first state variable, i.e. the beam deflection at the point $x$ and the fixed $t$. The LQ problem, with the finite time horizon $T$, lies in minimization of the (quadratic cost) functional

$$
J\left(\boldsymbol{u}_{0}, w\right)=\|w\|_{L^{2}(0, T ; C)}^{2}+\|y\|_{L^{2}(0, T ; C)}^{2}
$$

over trajectories of Eq. (16).

This functional is not standard for LQ problem with finite time horizon; it is modified a little. For the standard LQ problem, the theory of control gives an answer how to construct an optimal regulator to ensure asymptotic stability closed loop system and the form of optimal control is also known. It will be the subject of a future research.

In the next sub-sections, the control is assumed in the form $f_{t}=\exp \left(\mathrm{i} \omega_{f} t\right) \in L_{l o c}^{2}(0, \infty ; C)$, where $C$ is the complex number set; note that $f_{t}$ is not optimal. For such $f_{t}$, the value of the functional is calculated. This value depends, among other, on actuators distribution. Looking for a minimum of this function one finds the optimal actuators distribution.

\section{Optimal distribution of $n_{a}$ actuators}

It is further assumed that:

- there are $n_{a}$ actuators with coordinates of their centers $\left\{x_{a}\right\}$, Fig. $2\left(a=1,2, \ldots, n_{a}\right)$;
- $f_{t}=\exp \left(\mathrm{i} \omega_{k} t\right)$, where $\omega_{k}$ is the angular excited frequency of excitation $\left(\omega_{f}=\omega_{k}\right)$;

- $q_{E}(x)=q_{0} X_{k}(x)$, where $q_{0}$ is the excitation amplitude and $X_{k}(x)$ is the excitation form; as a result of above assumptions, the beam vibrates only in $k$ mode.

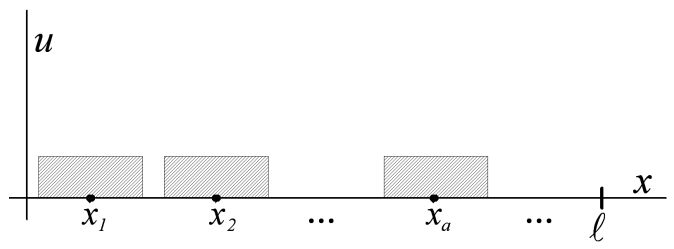

Fig. 2. Distribution of actuators.

In this case, $f_{x}$ is given by Eq. (9), whereas the function representing the first coordinate in Eq. (14) takes the form

$$
\begin{aligned}
& z_{1}(x, t)=C_{k} X_{k}(x) \exp \left(\mathrm{i} \omega_{k} t\right)=C_{k}^{*} \\
& \quad \times\left(-I_{q, k}+\sum_{a} p_{a} \kappa_{k}\left(x_{a}\right)\right) X_{k}(x) \exp \left(\mathrm{i} \omega_{k} t\right) .
\end{aligned}
$$

The functional for the control $f_{t}$ depends on distribution of actuators $\left\{x_{a}\right\}$ and has the form

$$
\begin{gathered}
J\left(x_{1}, \ldots, x_{n_{a}}\right)=\int_{0}^{T}\left|\exp \left(\mathrm{i} \omega_{k} t\right)\right|^{2} \mathrm{~d} t+\int_{0}^{T} \mid C_{k}^{*} \\
\times\left.\left(-I_{q, k}+\sum_{a} p_{a} \kappa_{k}\left(x_{a}\right)\right) X_{k}(x) \exp \left(\mathrm{i} \omega_{k} t\right)\right|^{2} \mathrm{~d} t= \\
T+\left(C_{k}^{*}\right)^{2}\left|-I_{q, k}+\sum_{a} p_{a} \kappa_{k}\left(x_{a}\right)\right|^{2} X_{k}^{2} T .
\end{gathered}
$$

Let us assume, that $-I_{q, k}+\sum_{a} p_{a} \kappa_{k}\left(x_{a}\right)<0$ [4]; otherwise, i.e. $-I_{q, k}+\sum_{a} p_{a} \kappa_{k}\left(x_{a}\right)>0$, instead of reduction of the vibrations, an increase would be obtained, so this case is not considered.

A mathematical problem appears: to find such distribution of actuators $\left\{x_{a}\right\}$ for which the value of functional $J$ takes a minimum. The sufficient condition for existence of the extremum of the problem can be expressed in the form

$$
\begin{aligned}
& D_{1} J\left(x_{1}, x_{2}, \ldots, x_{n_{a}}\right)=2\left(C_{k}^{*}\right)^{2} \\
& \quad \times\left(-I_{q, k}+\sum_{a} p_{a} \kappa_{k}\left(x_{a}\right)\right) p_{1} D_{1} \kappa_{k}\left(x_{1}\right) X_{k}^{2} T=0, \\
& D_{2} J\left(x_{1}, x_{2}, \ldots, x_{n_{a}}\right)=2\left(C_{k}^{*}\right)^{2} \\
& \quad \times\left(-I_{q, k}+\sum_{a} p_{a} \kappa_{k}\left(x_{a}\right)\right) p_{2} D_{2} \kappa_{k}\left(x_{2}\right) X_{k}^{2} T=0, \\
& \quad \vdots \\
& \quad D_{n_{a}} J\left(x_{1}, x_{2}, \ldots, x_{n_{a}}\right)=2\left(C_{k}^{*}\right)^{2}\left(-I_{q, k}\right. \\
& \left.\quad+\sum_{a} p_{a} \kappa_{k}\left(x_{a}\right)\right) p_{n_{a}} D_{n_{a}} \kappa_{k}\left(x_{n_{a}}\right) X_{k}^{2} T=0,
\end{aligned}
$$

where $D_{x_{1}}(\cdot)=D_{1}(\cdot), \ldots, D_{a}(\cdot)=D_{n_{a}}(\cdot)$ for simplicity. 
Assuming $-I_{q, k}+\sum_{a} p_{a} \kappa_{k}\left(x_{a}\right)<0$, the points in which an extremum may occur must satisfy conditions

$$
D_{1} \kappa_{k}\left(x_{1}\right)=0, D_{2} \kappa_{k}\left(x_{2}\right)=0, \cdots D_{n_{a}} \kappa_{k}\left(x_{n_{a}}\right)=0 .(24)
$$

To find the sufficient conditions of existence of an extremum, the matrix of second derivatives (Hessian $H$ ) ought to be taken into account. Let the necessary condition be satisfied at the point $P=\left(x_{1}, x_{2}, \ldots, x_{n_{a}}\right)$. Then the mixed derivatives at this point are equal to zero, i.e. $D_{a b}^{2} J\left(x_{1}, x_{2}, \ldots, x_{n_{a}}\right)=0, a, b=1,2, \ldots, n_{a}$, $a \neq b$. So the Hessian $H$ at point $P$ is a diagonal matrix $H=\operatorname{diag}\left[D_{a a}^{2} J(P)\right]_{a}, a=1,2, \ldots, n_{a}$.

Note that the second derivatives of the functional take the following explicit form:

$$
\begin{aligned}
& D_{a a}^{2} J\left(x_{1}, x_{2}, \ldots x_{n_{a}}\right)=2\left(C_{k}^{*}\right)^{2}\left[p_{a}^{2}\left(D_{a} \kappa_{k}\left(x_{a}\right)\right)^{2}\right. \\
& \left.\quad+\left(-I_{q, k}+\sum_{a} p_{a} \kappa_{k}\left(x_{a}\right)\right) p_{a} D_{a a}^{2} \kappa_{k}\left(x_{a}\right)\right] X_{k}^{2} T .
\end{aligned}
$$

The sufficient conditions for existing of a minimum of the functional at point $P$ are that the main minors are positive:

$$
\begin{gathered}
D_{11}^{2} J>0, \quad \operatorname{det}\left[\begin{array}{cc}
D_{11}^{2} J & 0 \\
0 & D_{22}^{2} J
\end{array}\right]>0, \cdots \\
\operatorname{det}\left[\begin{array}{ccc}
D_{11}^{2} J & 0 & \cdots \\
0 & D_{22}^{2} J & \cdots \\
\vdots & \vdots & D_{n_{a} n_{a}}^{2} J
\end{array}\right]>0 .
\end{gathered}
$$

Because of assumption made above in Eq. (24), from the condition $D_{11}^{2} J>0$ it follows that $D_{11}^{2} \kappa_{k}\left(x_{1}\right)<0$. Therefore the sufficient conditions for existence of a minimum at point $P$ take the form

$$
\begin{aligned}
& D_{11}^{2} \kappa_{k}\left(x_{1}\right)<0, D_{22}^{2} \kappa_{k}\left(x_{2}\right)<0, \cdots \\
& D_{n_{a} n_{a}}^{2} \kappa_{k}\left(x_{a}\right)<0 .
\end{aligned}
$$

The results are identical to ones obtained in [2], so objective of the present paper is achieved.

\section{Summary and conclusions}

It should be emphasized that, in general, the solution of eigenproblem in a Hilbert space is different from the solution given in subsection 2 , but form of the first component of the solution (eigenvector) is the same as in Eq. (6). Eigenvalues are always different but a connection can be found between new eigenvalues and these introduce in section 2 .

Furthermore, the point $\left\{x_{\max }\right\}$ at which the curvature has the highest value should be found, but it is possible for the explicit form of the mode; for more details, see $[2,4]$.

In the present paper, the quadratic cost functional is a little modified for LQ problem with a finite time hori- zon. For standard LQ problem, theory of control gives an answer how to construct optimal regulator to ensure asymptotic stability closed loop system and also the form of optimal control is known. It will be the subject of a future research. Furthermore, other cost functions will be considered.

The conclusions enumerated below are derived assuming that all actuators are the same and the beam vibrates only in separate mode. Taking into account the theoretical considerations on the grounds of the control theory (strictly speaking, on the grounds of the LQ problem), the following conclusions may be formulated:

- an optimal distribution of the actuators is in the sub-domains of the separate beam mode with the locally maximum curvatures;

- the above conclusion confirms the results obtained on pure mathematical way in the present author's own papers.

The similar research for beam vibration (not for separate modes) and also for actuators different with regard to their geometrical and technical properties, may be more useful. The studies on these problems are always carried on.

\section{References}

[1] A. Brański, M. Borkowski, S. Szela, Acta Phys. Pol. A 118, 17 (2010).

[2] A. Brański, G. Lipiński, Acta Phys. Pol. A 119, 936 (2011).

[3] A. Brański, in: Acoustic Waves, ch. 18, Intech, Rijeka 2011, p. 397.

[4] A. Brański, Acta Phys. Pol. A 123, 1123 (2013).

[5] M.S. Kozień, B. Koltowski, Acta Phys. Pol. A $\mathbf{1 1 9}$, 1005 (2011).

[6] J. Wiciak, Acoust. Eng. 13, 5 (2004), (in Polish).

[7] M.S. Kozień, J. Wiciak, Acta Phys. Pol. A 116, 348 (2009).

[8] M.S. Kozień, J. Wiciak, Archiv. Acoust. 33, 643 (2008).

[9] J. Wiciak, Europ. Phys. J. ST 154, 229 (2008).

[10] M.S. Kozień, Acta Phys. Pol. A 123, 6 (2013).

[11] I. Bruant, L. Gallimard, S. Nikoukar, J. S. V. 329, 1615 (2010).

[12] I. Bruant, G. Coffignal, F. Lene, M. Verge, J. S. V 243, 861 (2001).

[13] A. Brański, S. Szela, Archiv. Acoust. 33, 413 (2008).

[14] M. Weiss, Ph.D. thesis, University of Groningen, 1994.

[15] S.M. Yang, Y.J. Lee, Smart Mater. Struct. 2, 96 (1993). 